\section{Effects of mobile messenger counseling on case management success for individuals engaging in self-harm or suicide attempts who were discharged from emergency departments}

\author{
Ji Min Seong ${ }^{1}$, Youngsuk Cho ${ }^{1,2}$, Gyu Chong Cho' ${ }^{1}$ Jongshill Lee ${ }^{2}$, \\ In Young Kim ${ }^{2}$, Hongtaek $\mathrm{Seo}^{3}$, Jungmi $\mathrm{Kim}^{3}$ \\ 'Department of Emergency Medicine, Kangdong Sacred Heart Hospital, Hallym University College of \\ Medicine, Seoul, Korea \\ ${ }^{2}$ Department of Biomedical Engineering, Hanyang University College of Medicine, Seoul, Korea \\ ${ }^{3}$ Social Work Department, Kangdong Sacred Heart Hospital, Seoul, Korea
}

Objective Postdischarge case management for self-harm or suicide attempters often fails; therefore, this study aimed to investigate the effects of mobile messenger counseling (MMC) on the postdischarge case management results among this patient group.

Methods A retrospective analysis was done with data collected from March 2015 to February 2020 that included self-harm or suicide attempters who had visited a Korean emergency department and were discharged. If patients consented, postdischarge case management and MMC were conducted from March 2017. The primary outcome was the rate of successful case management, which reflects the patients either connecting to a local psychiatric healthcare center or undergoing a follow-up at a neuropsychiatric outpatient department at least once following discharge. Using univariate and multivariate logistic regression analyses, we evaluated MMC's effects on these patients' postdischarge case management.

Results Of 913 patients, 604 participated in this study. In terms of successful case management, the MMC group showed a significantly higher rate than the non-MMC one $(28.3 \%$ vs. $16.1 \%$, $P=0.001$ ). A multivariate analysis demonstrated that access to postdischarge MMC (odds ratio, 2.149; 95\% confidence interval, 1.357-3.403; $P=0.001$ ) and giving consent for case management while in the emergency department were significantly associated with successful case management (odds ratio, 8.917; 95\% confidence interval, 5.610-14.173; $\mathrm{P}<0.001$ ).

Conclusion The use of MMC for self-harm or suicide attempters is associated with higher case management success rates by increasing their chances of connecting to a psychiatric healthcare center or a neuropsychiatric outpatient department.

Keywords Suicide; Self-injurious behavior; Smartphone
elSSN: 2383-4625

Received: 12 October 2020

Revised: 5 November 2020

Accepted: 16 November 2020

Correspondence to: Youngsuk Cho Department of Emergency Medicine, Kangdong Sacred Heart Hospital, Hallym University College of Medicine, 150 Seongan-ro, Gangdong-gu, Seoul 05355, Korea

E-mail: faith2love@kdh.or.kr ORCID

https://orcid.org/0000-0002-7648-924X

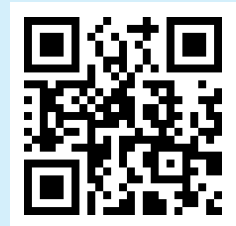

How to cite this article:

Seong JM, Cho Y, Cho GC, Lee J, Kim IY, Seo H, Kim J. Effects of mobile messenger counseling on case management success for individuals engaging in selfharm or suicide attempts who were discharged from emergency departments. Clin Exp Emerg Med 2021;8(1):48-54. https://doi.org/10.15441/ceem.20.133

This is an Open Access article distributed under the terms of the Creative Commons Attribution Non-Commercial License (https:// creativecommons.org/licenses/by-nc/4.0/). 


Capsule
Wumat is already known
Suicide is a major social problem, and self-harm or suicide attempters have a higher tendency to reattempt and are
more likely to succeed in the next suicide attempt. Therefore, it is important to manage self-harm or suicide attempters
even after discharge, but postdischarge case management often ends in failure.
What is new in the current study
$\begin{aligned} & \text { We conducted a retrospective study to explore the effect of mobile messenger counseling on postdischarge case man- } \\ & \text { agement. We found that the use of mobile messenger counseling for self-harm or suicide attempters is associated with } \\ & \text { higher post case management by increasing the chances of follow up with a local psychiatric healthcare center or a } \\ & \text { neuropsychiatric outpatient department. }\end{aligned}$

\section{INTRODUCTION}

Out of 6 million emergency department (ED) patients of South Korea, 33,451 were those who had attempted self-harm or suicide in 2018. ${ }^{1}$ As such, they accounted for $0.56 \%$ of the total ED patients, with this number showing an increasing trend in recent years. In 2019, 13,799 people killed themselves in South Korea, with it being noted that suicide was the leading cause of death in that year, particularly for those in their teens to their thirties. ${ }^{2}$ In addition, social expenditure caused by suicides was estimated at 5.6 billion dollars. ${ }^{3}$

Suicide became a particularly notable social problem in South Korea in 2009; since then, the local psychiatric healthcare centers have been providing postdischarge case management, with 52 selected hospitals conducting a national project to prevent selfharm and suicide attempts, based on the number of cases coming into their EDs. ${ }^{4}$ As it is reported that postdischarge case management for self-harm or suicide attempters reduces their reattempt rates, they are often advised to continue to undergo this followup management by visiting their local neuropsychiatric outpatient department (OPD) or by connecting to the local psychiatric healthcare center., ${ }^{5,6}$ Generally, because hospitalized self-harm or suicide attempters have a higher chance of coming into contact with physicians or social workers, they can often be fully managed and interviewed through face-to-face counseling. However, following discharge, case management often ends in failure as only telephonic or home-visit counseling is possible, making it difficult to persuade people to cooperate and receive continuous case management. ${ }^{7}$

To overcome these limitations, we aimed to identify a method to increase postdischarge case management's efficacy by using mobile messenger counseling (MMC) services. After taking into consideration the characteristics of mobile messengers that people use ubiquitously and therefore feel familiar with, we investi- gated the effects of MMC on the postdischarge case management results for patients with suicide attempts or self-harm.

\section{METHODS}

\section{Study design and subjects}

This was a retrospective analysis that investigated the period from March 2015 to February 2020 in a regional emergency center in Korea. We compared the postdischarge case management outcomes of patient who attempted self-harm or suicide on whether or not they had used the MMC. This emergency medical center operates through the use of a dedicated medical team for patients who have attempted self-harm or suicide. This team consists of emergency physicians, psychiatrists, and social workers as a part of a healthcare project entitled "Suicide prevention by enhancing Emergency Department based Post-suicide Attempt Case Management" that was created by the Korean Ministry of Health and Welfare. This study included patients who had visited this particular emergency medical center for self-harm or suicide attempts and who were then discharged from the ED. Those admitted to the hospital or those who could not use mobile messengers due to environmental factors such as imprisonment, were excluded. Additionally, any duplicated or incomplete data were excluded (Fig. 1). This study was approved by the institutional review board of Kangdong Sacred Heart Hospital (KDIRB-No. 201806-008). Informed consent was waived because of the retrospective nature of the study.

\section{Case management protocol using MMC}

Protocols were developed for self-harm or suicide attempters who had visited the ED. The protocols for this process were as follows: When a person visited the ED for these reasons, the emergency physicians performed the initial evaluation and medical treatment. Simultaneously, the psychiatrists and social work- 
913 Enrolled patients with self-harm or suicide attempts in the ED

309 Excluded subjects 294 Subjects admitted to hospital 9 Environmental factors (e.g., imprisonment) 6 Incomplete, duplicated data, etc.

604 Eligible patients

304 Subjects without mobile messenger counseling (non-MMC group)

Fig. 1. Flowchart of the study sample. ED, emergency department. MMC, mobile messenger counseling.

ers would intervene in each case via psychiatric interviews and counseling. If the patient consented to undergo case management, the social worker team then provided face-to-face or telephonic counseling over four weeks, regardless of whether the patient was hospitalized or discharged.

In addition, the postdischarge MMC was then added to the aforementioned protocol since March 2017. After the introduction of the MMC, regardless of whether consent was given to perform case management, patients were asked to undertake the postdischarge MMC. For patients who agreed to receive MMC, while considering all relevant privacy and security regulations, the social worker team created a one-to-one closed chat room for the counseling of each patient using KakaoTalk mobile messenger (Kakao Corp., Seoul, Korea). These chat rooms aimed to enable communication with any patients who were reluctant to receive face-toface or telephonic counseling; they were open to text messages 24/7 whenever the patient wanted to communicate and were then deleted after each person's case management ended.

\section{Data source and the outcome variables}

We used the case management database of our hospital, which is collected annually for the national suicide prevention project. All final case management results are processed anonymously and are coded with a series of identifier numbers that are only known by the data management personnel in order to minimize any bias. The independent variables in this study were participants' sex, age, self-harm or suicide attempt methods, whether patients consented to undergo case management or not, hospitalization, and postdischarge MMC. The age variable was categorized into three groups: underage ( $\leq 17$ years), youth/young people (18-65 years), and then middle-aged, elderly/senior, long-lived elderly ( $\geq 66$ years). ${ }^{8}$ The types of self-harm or suicide were divided into seven categories using information from the International Classification of External Causes of Injuries. ${ }^{9}$ The primary outcome of concern was the successful case management rate, which reflects whether the patient connected with a local psychiatric healthcare center or underwent follow-up at a neuropsychiatric OPD at least once after discharge from the ED.

\section{Statistical analysis}

We divided the patients into two groups: those who did not use MMC (the non-MMC group) and those that did use it (MMC group), and then compared the main outcome between them. All patients who visited the ED before the introduction of MMC were classified into the non-MMC group, with those who had visited the ED after the introduction of MMC then being classified into one of two groups (the non-MMC vs. the MMC group) based on if they gave consent to undergo MMC. All the categorical variables were expressed as frequencies and percentages, while continuous variables were expressed as median values and quartiles. Parametric methods, such as an independent t-test or chi-square tests, were used if normality was satisfied. If not, non-parametric methods, such as a Mann-Whitney U-test or chi-square tests, were used.

Moreover, we analyzed the outcome according to the independent variables in the univariate analysis. Thereafter, a multivariate logistic regression analysis, with the backward elimination method, was performed to evaluate the effects of MMC; specifically, whether it influences the successful case management or not of these patients after adjusting for other confounders. Successful case management was set as the dependent variable, while sex, age, self-harm/suicide methods, postdischarge MMC, and consent to undergo case management given while in the ED were all set as the covariates. To determine whether the logistic model fits the data, we calculated the Hosmer-Lemeshow goodness of fit. The results were calculated as odds ratios (ORs) and 95\% confidence intervals (Cls). For all statistical analyses, PASW Statistics ver. 18.0 (SPSS Inc., Chicago, IL, USA) was used, with the statistical significance level being defined as $\mathrm{P}<0.05$.

\section{RESULTS}

A total of 913 self-harm or suicide attempters visited the emergency medical center from March 2015 to February 2020. After 
excluding 309 people, 604 were then included in this study. The reasons for the exclusions were because 294 were admitted to the hospital; nine could not use mobile messengers due to reasons such as imprisonment; and, finally, six were excluded due to either incomplete or duplicated data, etc. This resulted in 304 people being in the non-MMC group and 300 people in the MMC group (Fig. 1).

There was a statistical difference found in the number of females between the non-MMC and MMC groups (66.1\% vs. 76.3\%, $P=0.006$ ). The median age and interquartile range was 38.7 years (25.0-50.3) in the non-MMC group and 44.0 years (20.0-46.8) in the MMC group. The median age was higher in the latter, with there being a statistical difference found between the two groups in this variable $(P<0.001)$. Moreover, there was a statistical difference found in the age groups, resulting in 17 underage (5.6\%), 268 young people (88.2\%), and 19 elderly/senior (6.2\%) people in the non-MMC group, and 35 underage (11.7\%), 253 young people (84.3\%), 12 elderly/senior (4.0\%) people in the MMC group $(P=0.016)$. Types of self-harm or suicide methods in the nonMMC group were as follows: 129 cases (42.4\%) involving poisoning, 123 cases (40.5\%) involving cutting/piercing/stabbing, 14 cases (4.6\%) involving hanging/strangling, 13 cases (4.3\%) involving drowning/near-drowning/submersion, 13 cases (4.3\%) involving smoke inhalation, and nine cases (3.0\%) involving falling/jumping. The methods utilized in the MMC group were as follows: 150 cases (50.0\%) involving poisoning, 126 cases (42.0\%)

Table 1. Basic characteristics of the self-harm or suicide attempt patients $(n=604)$

\begin{tabular}{|c|c|c|c|}
\hline & $\begin{array}{c}\text { Non-MMC } \\
\text { group } \\
(n=304)\end{array}$ & $\begin{array}{c}\text { MMC group } \\
(n=300)\end{array}$ & P-value \\
\hline Sex, female & $201(66.1)$ & $229(76.3)$ & 0.006 \\
\hline Age (yr) & $38.7(25.0-50.3)$ & $44.0(20.0-46.8)$ & $<0.001$ \\
\hline Age group (yr) & & & 0.016 \\
\hline$\leq 17$ & $17(5.6)$ & $35(11.7)$ & \\
\hline $18-65$ & $268(88.2)$ & $253(84.3)$ & \\
\hline$\geq 66$ & $19(6.2)$ & $12(4.0)$ & \\
\hline Self-harm or suicide methods & & & 0.018 \\
\hline Poisoning & $129(42.4)$ & $150(50.0)$ & \\
\hline Cut/pierce/stabbing & $123(40.5)$ & $126(42.0)$ & \\
\hline Hanging/strangling & $14(4.6)$ & $6(2.0)$ & \\
\hline Drowning/submersion & $13(4.3)$ & $4(1.3)$ & \\
\hline Smoke inhalation & $13(4.3)$ & $4(1.3)$ & \\
\hline Falling/jumping & $9(3.0)$ & $9(3.0)$ & \\
\hline Other specified mechanism & $3(1.0)$ & $1(0.3)$ & \\
\hline Consent to case management in ED & $111(36.6)$ & $110(36.8)$ & 0.968 \\
\hline
\end{tabular}

Values are presented as number (\%) or median (interquartile range). $M M C$, mobile messenger counseling; ED, emergency department. involving cutting/piercing/stabbing, six cases (2.0\%) involving hanging/strangling, four cases (1.3\%) involving drowning/neardrowning/submersion, four cases (1.3\%) involving smoke inhalation, and nine cases (3.0\%) involving falling/jumping. There was a statistical difference found between the two groups $(P=0.018)$ in terms of their suicide methods. However, there was no significant difference observed between the two groups in their consent rates for undergoing primary case management (36.6\% vs. $36.8 \%$, $\mathrm{P}=0.968$ ) (Table 1).

The rate of patients who connected with their local psychiatric healthcare center showed a significant difference between the MMC and non-MMC groups ( $11.7 \%$ vs. $5.9 \%, P=0.013$ ). Additionally, the MMC group showed a significantly higher rate of engaging in follow-ups at a neuropsychiatric OPD (25.0\% vs. $12.8 \%, P<0.001)$. In terms of successful case management, the MMC group again showed a significantly higher rate than did the non-MMC one (28.3\% vs. $16.1 \%, P=0.001)$.

In the univariate analysis, the rate of female patients in the success group showed statistically higher ( $80.6 \%$ vs. $68.5 \%, P=0.006$ ), and those of the 18-65 age patients showed lower than the other group $(76.9 \%$ vs. $88.9 \%, \mathrm{P}=0.027)$, both showed statistically higher success rates than did the other groups. Conversely, there was no significant difference observed among the various selfharm or suicide methods utilized $(P=0.753)$. The postdischarge

Table 2. Univariate analysis of the self-harm or suicide attempt patients in terms of successful case management $(n=604)$

\begin{tabular}{lccc}
\hline & $\begin{array}{c}\text { No success } \\
(\mathrm{n}=470)\end{array}$ & $\begin{array}{c}\text { Success } \\
(\mathrm{n}=134)\end{array}$ & P-value \\
\hline $\begin{array}{l}\text { Sex, Female } \\
\text { Age (yr) }\end{array}$ & $322(68.5)$ & $108(80.6)$ & 0.006 \\
$\leq 17$ & $30(6.4)$ & $22(16.4)$ & 0.027 \\
$18-65$ & $418(88.9)$ & $103(76.9)$ & \\
$\geq 66$ & $22(4.7)$ & $9(6.7)$ & \\
Self-harm or suicide methods & & & \\
Poisoning & $206(43.8)$ & $73(54.5)$ & 0.753 \\
Cut/pierce/stabbing & $204(43.4)$ & $45(33.6)$ & \\
Hanging/strangling & $15(3.2)$ & $5(3.7)$ & \\
Drowning/submersion & $15(3.2)$ & $2(1.5)$ & \\
Smoke inhalation & $15(3.2)$ & $2(1.5)$ & \\
Falling/jumping & $12(2.6)$ & $6(4.5)$ & \\
$\quad$ Other specified mechanism & $3(0.6)$ & $1(0.7)$ & \\
Postdischarge MMC & & & \\
No & $255(54.3)$ & $49(36.6)$ & $<0.001$ \\
Yes & $215(45.7)$ & $85(63.4)$ & \\
Consent to case management in ED & & & \\
No & $347(74.1)$ & $34(25.4)$ & $<0.001$ \\
Yes & $121(25.9)$ & $100(74.6)$ & \\
\hline
\end{tabular}

Values are presented as numbers (\%).

$M M C$, mobile messenger counseling; $E D$, emergency department. 
Table 3. Multivariate analysis of the self-harm or suicide attempt patients in terms of successful case management ( $n=604)$

\begin{tabular}{|c|c|c|c|c|c|c|}
\hline Variable & Crude OR & $95 \% \mathrm{Cl}$ & P-value & Adjusted OR & $95 \% \mathrm{Cl}$ & P-value \\
\hline Sex, female ${ }^{a)}$ & 1.909 & $1.193-3.056$ & 0.007 & 2.168 & $1.250-3.761$ & 0.006 \\
\hline Age group $(y r)^{a)}$ & & & 0.001 & & & 0.053 \\
\hline$\leq 17$ & - & - & - & - & - & - \\
\hline $18-65$ & 0.336 & $0.186-0.607$ & $<0.001$ & 0.473 & $0.232-0.966$ & 0.040 \\
\hline$\geq 66$ & 0.558 & $0.216-1.444$ & 0.229 & 0.914 & $0.285-2.932$ & 0.880 \\
\hline Self-harm/suicide methods & & & 0.215 & & & 0.812 \\
\hline Poisoning & - & - & - & - & - & - \\
\hline Cut/pierce/stabbing & 0.622 & $0.409-0.946$ & 0.027 & 0.681 & $0.418-1.111$ & 0.124 \\
\hline Hanging/strangling & 0.941 & $0.330-2.679$ & 0.909 & 1.001 & $0.311-3.221$ & 0.999 \\
\hline Drowning/submersion & 0.376 & $0.084-1.685$ & 0.201 & 0.715 & $0.138-3.713$ & 0.690 \\
\hline Smoke inhalation & 0.376 & $0.084-1.685$ & 0.201 & 0.953 & $0.184-4.925$ & 0.954 \\
\hline Falling/jumping & 1.411 & $0.511-3.896$ & 0.506 & 1.235 & $0.379-4.024$ & 0.726 \\
\hline Other mechanism & 0.941 & $0.096-9.186$ & 0.958 & 0.619 & $0.052-7.293$ & 0.703 \\
\hline Postdischarge $\mathrm{MMC}^{\text {a) }}$ & 2.057 & $1.385-3.056$ & $<0.001$ & 2.149 & $1.357-3.403$ & 0.001 \\
\hline Consent to case management in $E D^{a)}$ & 8.483 & $5.460-13.180$ & $<0.001$ & 8.917 & $5.610-14.173$ & $<0.001$ \\
\hline
\end{tabular}

$M M C$, mobile messenger counseling; $\mathrm{OR}$, odds ratio; $\mathrm{Cl}$, confidence interval; $\mathrm{ED}$, emergency department.

${ }^{a}$ Variables selected via the backward elimination method.

MMC and consent given to undergo case management while in the ED showed a statistically significant difference in terms of successful case management ( $63.4 \%$ vs. $45.7 \%, P<0.001$ and $74.6 \%$ vs. $25.9 \%, P<0.001$, respectively) (Table 2 ). In the multivariate analysis, age and self-harm or suicide methods did not show any significant difference between the two groups $(P=0.053$ and $P=0.812$ ). However, the multivariate analysis did demonstrate that being female $(\mathrm{OR}, 2.168 ; 95 \% \mathrm{Cl}, 1.250-3.761 ; \mathrm{P}=0.006)$, receiving postdischarge $M M C\left(O R, 2.149 ; 95 \% \mathrm{Cl}_{1} 1.357-3.403\right.$; $P=0.001)$, and giving one's consent to undergo case management in the ED all showed statistically significant differences between the two groups $(\mathrm{OR}, 8.917 ; 95 \% \mathrm{Cl}, 5.610-14.173 ; \mathrm{P}<0.001)$ (Table 3). The Hosmer-Lemeshow goodness-of-fit test was 0.332.

\section{DISCUSSION}

The MMC group included higher numbers of females, younger individuals, and patients who had attempted self-harm or suicide by poisoning. The most influential factor associated with successful case management was whether the patients had consented to undergo case management while in $\mathrm{ED}(\mathrm{OR}, 8.917 ; 95 \% \mathrm{Cl}, 5.610-$ 14.173; $P<0.001)$. However, if the patients did consent to case management while within the ED, this might mean that they were more open-minded to receiving counseling in the first place, meaning that the results could have been natural. Therefore, postdischarge MMC is likely a clinically significant factor for ensuring favorable clinical and treatment outcomes (OR, 2.149;95\% Cl, 1.357-3.403; $P=0.001$ ).

It is known that self-harm or suicide attempts pose a serious threat to life and are highly likely to be accompanied by other serious psychiatric problems. ${ }^{10}$ Additionally, because most self-harm or suicide attempters visit EDs for treatment, these events should be considered psychiatric emergencies requiring immediate intervention from psychiatrists, in addition to medical treatments from emergency physicians. ${ }^{11-14}$ Even after discharge from the ED, if self-harm or suicide attempters are unable to resolve their living situations or psychiatric problems, they may engage in reattempts. Because self-harm or suicide attempters have a higher tendency of reattempting suicide and are more likely to succeed in their next attempt, ${ }^{14}$ many studies have been conducted aimed at reducing the suicide attempt rate, as well as to identify factors that affect it. ${ }^{5,6,15-17} \mathrm{~A}$ previous study found that there was a decreased reattempt rate within six months when patients had visited a neuropsychiatric OPD.,6 Similarly, another study conducted in 2012 reported that continuous case management of suicide or self-harm attempters, through connecting them to local psychiatric healthcare centers, could reduce their reattempt rate. ${ }^{5,6}$ However, in general, as a very low percentage of these patients, especially those who have been discharged, usually agree to case management in the ED, the effect of these interventions is limited in practice. Although it may vary depending on each study, most researches reported that $5.4 \%$ to $20.2 \%$ of patients were connected to local psychiatric healthcare centers. ${ }^{5,15,16}$ Another study reported that $35.1 \%$ of self-harm or suicide attempters revisited neuropsychiatric OPDs. ${ }^{17}$ In this study, consistent with previous research, only 8.7\% (53/604) and 18.9\% (114/604) (Table 4) of patients continued treatment through either connecting to local psychiatric healthcare centers or a follow-up to neuropsy- 
Table 4. Comparison of the two groups according to the presence of postdischarge mobile messenger counseling $(n=604)$

\begin{tabular}{lccc}
\hline & $\begin{array}{c}\text { Non-MMC group } \\
(\mathrm{n}=304)\end{array}$ & $\begin{array}{c}\text { MMC group } \\
(\mathrm{n}=300)\end{array}$ & P-value \\
\hline $\begin{array}{c}\text { Connecting to local } \\
\text { community center }\end{array}$ & $18(5.9)$ & $35(11.7)$ & 0.013 \\
$\begin{array}{c}\text { Follow-up to } \\
\text { neuropsychiatric OPD }\end{array}$ & $39(12.8)$ & $75(25.0)$ & $<0.001$ \\
$\begin{array}{c}\text { Successful case } \\
\text { management }\end{array}$ & $49(16.1)$ & $85(28.3)$ & 0.001 \\
\hline
\end{tabular}

Values are presented as numbers (\%).

$\mathrm{MMC}$, mobile messenger counseling; $\mathrm{OPD}$, outpatient department.

${ }^{a}$ Successful case management means the number of cases that were transferred to a local community center or engaged in a follow-up at a neuropsychiatric OPD.

chiatric OPD, respectively. Therefore, it is important to encourage patients to continue treatment at a local psychiatric healthcare center or through visiting a neuropsychiatric OPD; however, there has not been much interest shown in this approach within the field of emergency medicine yet.

As the number of smartphone users is increasing, mobile commercialization has spread to the medical field as well, with the development of more than 325,000 health-based applications in the United States alone. ${ }^{18}$ Recent studies have reported on mobile applications' effectiveness in reducing depression and psychological distress. ${ }^{19}$ In addition to mobile applications, communication through mobile messengers is widely conducted in people's daily lives. One survey conducted in South Korea showed that the frequency of using mobile messengers as a simple communication tool accounted for $44.9 \%$, which by far surpassed phone calls (38.1\%) or text messages $(17.0 \%) .{ }^{20}$ Smartphone users prefer mobile messengers because of their convenience, familiarity, and decreased psychological burden. These advantages give users greater initiatives in self-expression and in the formation of their social relations, thus positively influencing their relationships and thereby providing them with a greater sense of happiness. Through permanently open chat rooms, people can maintain constant social relations, communicate whenever they want, and express themselves more effectively through the use of various emoticons and media such as photos. ${ }^{21,22}$ Considering these characteristics of mobile messengers, this study investigated the effect of mobile messengers as a counseling tool for self-harm or suicide attempters following discharge from the ED. According to our findings, the addition of mobile messenger apps in counseling results in a significantly higher case management success rate, which may be ascribed to the aforementioned characteristics of mobile messengers as a communication tool..$^{23,24}$ Given this, if emergency physicians are able to conduct case management using mobile messengers, and they recommend using it to their patients, it can improve case management success rates following discharge by maintaining a minimal window of communication.

This study does possess a few limitations. First, it was a smallsized study conducted at a single hospital in Korea. Because it is impossible to include the characteristics of the patient group completely, and due to the possible impacts of regional and social environments, it might be difficult to generalize these results. It is especially challenging to adjust variables such as patients' socioeconomic levels and their histories of psychiatric illnesses. Additionally, although this study noted that the logistic regression model fit the data well, its ability to predict the dependent variable using the independent variables was relatively low $\left(R^{2}=\right.$ 0.313 ). This means that many uninvestigated variables could have been affecting the success of the case management; further studies are therefore needed in this regard. Moreover, unmeasurable confounders, such as the social workers' improved competencies over time and their expectations of achieving better outcomes by utilizing the MMC, could not be assessed in this research. Despite the introduction of the MMC, the experiences of the elderly group, who were unfamiliar with smartphone use, as well as people who were unable to use it for various reasons, might have been excluded in this study. Furthermore, because the data covered only whether the MMC was performed or not, the difference in each patient's response and participation level could not be considered. As this study compared only the patients' case management success rates, it is difficult to ascertain whether the MMC could ultimately decrease future suicide or self-harm reattempt rates. In the future, large-scale multicenter prospective studies are needed to evaluate the effects of the MMC for discharged patients.

In conclusion, we found that the use of mobile messengers for counseling self-harm or suicide attempters leads to higher case management success rates by increasing their likelihood of connecting to a local psychiatric healthcare center or a neuropsychiatric OPD. It is believed that if emergency physicians, psychiatrists, and social services workers make efforts to continue communicating with patients through mobile messengers following discharge, these apps can be used as an effective alternative treatment method to reduce patients reattempting self-harm or suicidal behaviors.

\section{CONFLICT OF INTEREST}

No potential conflict of interest relevant to this article was reported. 


\section{REFERENCES}

1. National Emergency Medical Center. 2019 Annual report of National Emergency Department Information System [Internet]. Seoul: National Emergency Medical Center; 2019 [cited 2020 Jan 2]. Available from: https://www.e-gen.or.kr/nemc/ statistics_annual_report.do?brdclscd $=02$.

2. Statistics Korea. Cause of death [Internet]. Daejeon: Korean Statistical Information Service; 2018 [cited 2018 Mar 25]. Available from: https://kosis.kr/statisticsList/statisticsListlndex.do?menuld = M_01_01\&tvwcd = MT_ZTITLECtparmTabld = M_01_01CtoutLink= YctentrType $=$ \#content-group.

3. National Health Insurance Service. Analysis of the social costs of major diseases for setting health care policy priorities [Internet]. Seoul: National Health Insurance Service; 2014 [cited 2018 Mar 25]. Available from: https://www.nhis.or.kr/nhis/together $/$ wbhaec07800m01.do? mode $=$ viewctarticleNo $=112970$.

4. Ministry of Health and Welfare. National action plan for suicide prevention [Internet]. Sejong: Ministry of Health and Welfare; 2018 [cited 2018 Mar 25]. Available from: http://www. mohw.go.kr/react/al/sal0301vw.jsp?PAR_MENU_ID = 04EtMENU _ID = 0403\&CONT_SEQ $=343649$.

5. Jang N, Kim J, Park WB, et al. Efficacy of a program associated with a local community of suicide attempters who visited a regional emergency medical center. J Korean Soc Emerg Med 2012;23:696-704.

6. Kapur N, Cooper J, Hiroeh U, May C, Appleby L, House A. Emergency department management and outcome for self-poisoning: a cohort study. Gen Hosp Psychiatry 2004;26:36-41.

7. Korea Suicide Prevention Center. Crisis control protocol for suicide and psychiatric disease [Internet]. Seoul: Korea Suicide Prevention Center; 2013 [cited 2018 Mar 25]. Available from: http://www.spckorea.or.kr/sub.php?id = pub\&tmode = view\&tmen ukey $=9$ \&tsrhctgr $=$ subject\&tsrhstr $=\% \mathrm{ED} \% 94 \% 84 \% \mathrm{~EB} \% \mathrm{~A} 1 \% 9$ C\%ED\%86\%A0\%EC\%BD\%9CCtoptr $=$ ctidx $=21 \mathrm{ctpage}=1 \mathrm{ctme}$ nukey $=9$.

8. Woo S, Lee SW, Lee K, et al. Characteristics of high-intent suicide attempters admitted to emergency departments. J Korean Med Sci 2018;33:e259.

9. ICECI Coordination and Maintenance Group. International Classification of External Causes of Injuries (ICECl) version 1.2 p.47-50 [Internet]. Amsterdam: Consumer Safety Institute; 2014 [cited 2020 Dec 10]. Available from: https://www.whofic.nl/sites/default/files/2018-05/ICECl\%20in\%20English.pdf.

10. Roy A. Suicide. In: Sadock BJ, Sadock VA, editors. Comprehen- sive textbook of psychiatry. 7th ed. Philadelphia, PA: Lippincott Williams \& Wilkins; 2000. p.2031-40.

11. Gi YG, Kim EJ, Park HU, Kang SH, Seo JH. A study of medical services for psychiatric emergency patients. Seoul: National Center for Mental Health; 2013.

12. Park JH, Wi DH. Clinical analysis of the suicidal attempters visiting emergency center. J Korean Soc Emerg Med 1999;10: 568-78.

13. Sudarsanan S, Chaudhury S, Pawar AA, Salujha SK, Srivastava K. Psychiatric emergencies. Med J Armed Forces India 2004; 60:59-62.

14. Harris EC, Barraclough B. Suicide as an outcome for mental disorders: a meta-analysis. Br J Psychiatry 1997;170:205-28.

15. Lee J, Kang HG, Kim C, et al. The factors affecting the registration rates for emergency department based post-suicidal care program. J Korean Soc Clin Toxicol 2015;13:25-32.

16. Kim DK, Chun BJ, Moon JM, et al. Contributing factors for the registration rates within emergency department based postsuicidal care program. J Korean Soc Clin Toxicol 2016;14:54-9.

17. Lee JH, Yang SJ, Eun SW, Jin SC, Choi WI, Jung SW. Limitation of psychiatric intervention for suicidal drug intoxication patients in emergency room. J Korean Soc Clin Toxicol 2016;14: 37-46.

18. Bates DW, Landman A, Levine DM. Health apps and health policy: what is needed? JAMA 2018;320:1975-6.

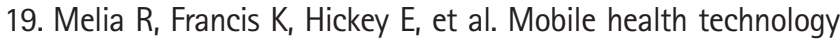
interventions for suicide prevention: systematic review. JMIR mHealth uHealth 2020;8:e12516.

20. Macromillembrain. 2018 Research for use of smart-phone and perception about phone call [Internet]. Seoul: Macromillembrain; 2018 [cited 2018 Aug 29]. Available from: https:// trendmonitor.co.kr/tmweb/trend/allTrend/detail.do?bldx $=167$ 9\&tcode $=0401 \&$ trendType $=$ CKOREA .

21. Lee Jl. A study on the college students' use and daily experience of mobile instant messenger. Media Gend Cult 2014;29:37-70.

22. Kim HJ, Kim MS, Oh DG, Kim ST. The effects of smartphone use on interpersonal relationship and happiness. J Cybercommunication Acad Soc 2014;31:163-98.

23. Krynski L, Goldfarb G, Maglio I. Technology-mediated communication with patients: WhatsApp Messenger, e-mail, patient portals: a challenge for pediatricians in the digital era. Arch Argent Pediatr 2018;116:e554-9.

24. Jeon SC, Kim JH, Kim SJ, et al. Effect of sending educational video clips via smartphone mobile messenger on bowel preparation before colonoscopy. Clin Endosc 2019;52:53-8. 